\title{
Diabetic Nephropathy: a Tangled Web to Unweave
}

\author{
Corey Magee $^{1} \cdot$ David J. Grieve $^{1} \cdot$ Chris J. Watson ${ }^{1} \cdot$ Derek P. Brazil $^{1}$
}

Published online: 27 September 2017

(C) The Author(s) 2017. This article is an open access publication

\begin{abstract}
Diabetic nephropathy (DN) is currently the leading cause of end-stage renal disease globally. Given the increasing incidence of diabetes, many experts hold the view that DN will eventually progress toward pandemic proportions. Whilst hyperglycaemia-induced vascular dysfunction is the primary initiating mechanism in $\mathrm{DN}$, its progression is also driven by a heterogeneous set of pathological mechanisms, including oxidative stress, inflammation and fibrosis. Current treatment strategies for DN are targeted against the fundamental dysregulation of glycaemia and hypertension. Unfortunately, these standards of care can delay but do not prevent disease progression or the significant emotional, physical and financial costs associated with this disease. As such, there is a pressing need to develop novel therapeutics that are both effective and safe. Set against the genomic era, numerous potential target pathways in DN have been identified. However, the clinical translation of basic DN research has been met with a number of challenges. Moreover, the notion of DN as a purely vascular disease is outdated and it has become clear that $\mathrm{DN}$ is a multi-dimensional, multi-cellular condition. The review will highlight the current therapeutic approaches for DN and provide an insight into how the inherent complexity of DN is shaping the research pathways toward the development and clinical translation of novel therapeutic strategies.
\end{abstract}

David J. Grieve

d.grieve@qub.ac.uk

1 Centre for Experimental Medicine, Queen's University Belfast, Wellcome-Wolfson Building, 97 Lisburn Road, Belfast, Northern Ireland BT9 7AE, UK
Keywords Diabetic nephropathy · Vascular dysfunction · Renin-angiotensin-aldosterone system · Oxidative stress · Inflammation · Novel therapies

\section{Introduction}

Diabetes mellitus (DM) is a systemic disease that is characterised by an inability of the body to either produce or effectively respond to the glucose-regulating hormone, insulin [1]. Type $1 \mathrm{DM}$ results from an idiopathic autoimmune destruction of pancreatic $\beta$-cells, whilst widespread peripheral insulin resistance drives type $2 \mathrm{DM}$ [1]. The resulting hyperglycaemia upsets haemodynamic and metabolic homeostases, whilst the chronic nature of this microenvironmental disequilibrium promotes the development of diffuse cellular abnormalities. As a result, many patients develop serious complications, the most devastating of which is extensive vascular dysfunction [2]. Given that the kidneys are highly sensitive to both haemodynamic and metabolic alterations, these organs are vulnerable targets within the diabetic milieu. Diabetic nephropathy (DN) is a key microvascular complication of DM. $\mathrm{DN}$ is characterised by albuminuria (urinary albumin to creatinine ratio $\geq 30 \mathrm{mg} / \mathrm{g}$ ) and an eventual decline in the estimated glomerular filtration rate $\left(e G F R<60 \mathrm{ml} / \mathrm{min} / 1.73 \mathrm{~m}^{2}\right)$ [3] $\mathrm{DN}$ follows distinct phases, where glomerular hyperfiltration is followed by a relentless decline in renal function, typically occurring over a 15-20 year period.

Currently, DN is the leading cause of end-stage renal disease (ESRD) globally [3]. Whilst the natural history of DN can vary slightly, patients often experience a chronic condition where progressive structural changes in the kidney correlate with various stages of clinical renal deterioration. The development of ESRD requires the use of dialysis or renal transplantation, both of which can be associated with excess 
morbidity and mortality $[4,5]$. Moreover, DN incidence rates show no signs of slowing. In the USA alone, $42 \%$ of all ESRD cases had a diagnosis of DN [6]. The importance of maintaining optimal renal function is further evidenced by the fact that $\mathrm{DN}$ is responsible for the vast proportion of excess mortality risk in patients with DM [7, 8]. Thus, there is a clear unmet need to develop novel DN-targeted therapeutics that can curtail the unrelenting progression of this disease and, hence, improve both the quality and quantity of life for this patient group. This review will highlight the key molecular mechanisms that drive $\mathrm{DN}$ whilst stressing the need to view $\mathrm{DN}$ as more than a simple vascular complication. More importantly, it will provide a critical insight into a range of exciting new DN treatment strategies that are on the horizon.

\section{Diabetic Nephropathy Pathogenesis}

\section{Metabolic and Haemodynamic Dysregulations}

DN pathogenesis is characterised by a complex interplay between metabolic and haemodynamic disruptions [9]. In DN, hyperglycaemia-induced metabolic impairment is central to the development and progression of DN (Fig. 1) and, hence, the appearance of defining renal lesions [10]. These include glomerular basement membrane thickening, mesangial expansion and the appearance of characteristic Kimmelstein-Wilson nodules. These changes occur secondary to hyperglycaemiainduced oxidative stress and accumulation of advanced glycation end products. Additional critical mediators of DN include haemodynamic elements, such as activation of vasoactive hormonal pathways, most commonly the reninangiotensin-aldosterone system (RAAS). The associated systemic hypertension contributes to increased intra-glomerular pressure [8]. Glomerular microvascular lesions develop secondary to altered blood flow and increased vascular permeability. The underlying signalling pathways involved are highly complex, and current research is intensively focused on a range of key molecular drivers.

Central to aberrant signalling in DN is the transforming growth factor-beta 1 (TGF- $\beta_{1}$ ), which has long been recognised as a key driver of DN [11]. TGF- $\beta_{1}$ expression is elevated within various constituent kidney cells in DN [12, 13]. Dysfunctional TGF- $\beta_{1}$ signalling modulates glucose flux into renal cells by upregulating the glucose transporter, GLUT-1 [10]. In effect, TGF- $\beta_{1}$ is at the centre of a complex signalling cascade-driving DN [14]. SMAD proteins are the intra-cellular signalling effectors of TGF- $\beta_{1}$ secondary to their ability to translocate to the nucleus and regulate transcription [15]. Animal models have highlighted the importance of the TGF- $\beta$ /SMAD pathway in the development of diabetic glomerulosclerosis and tubulointerstitial fibrosis [16, 17]. Beyond this canonical pathway, recent studies have proven the existence of complex crosstalk between TGF- $\beta$ and other well-known SMAD-independent pathways, such as Src protooncogene non-receptor tyrosine kinase (Src) signalling. Moreover, this redox-driven crosstalk has been shown to upregulate pro-fibrotic markers [18] and may provide new opportunities for targeted intervention.

Aside from the TGF- $\beta$ pathway, a host of newly implicated genes has been identified, due in part to the upsurge in genome-wide association studies (GWAS). These have proven to be powerful tools for defining the genetic architecture of $\mathrm{DN}$ in patients. However, limitations of the genome-wide approach were recently exemplified by the GENIE consortium [19]. Despite being the largest DN-focused GWAS to date, this study highlighted the vast complexity underlying DN heritability. It is also important to realise that many newly
Fig. 1 The central role of hyperglycaemia in the development and progression of diabetic nephropathy

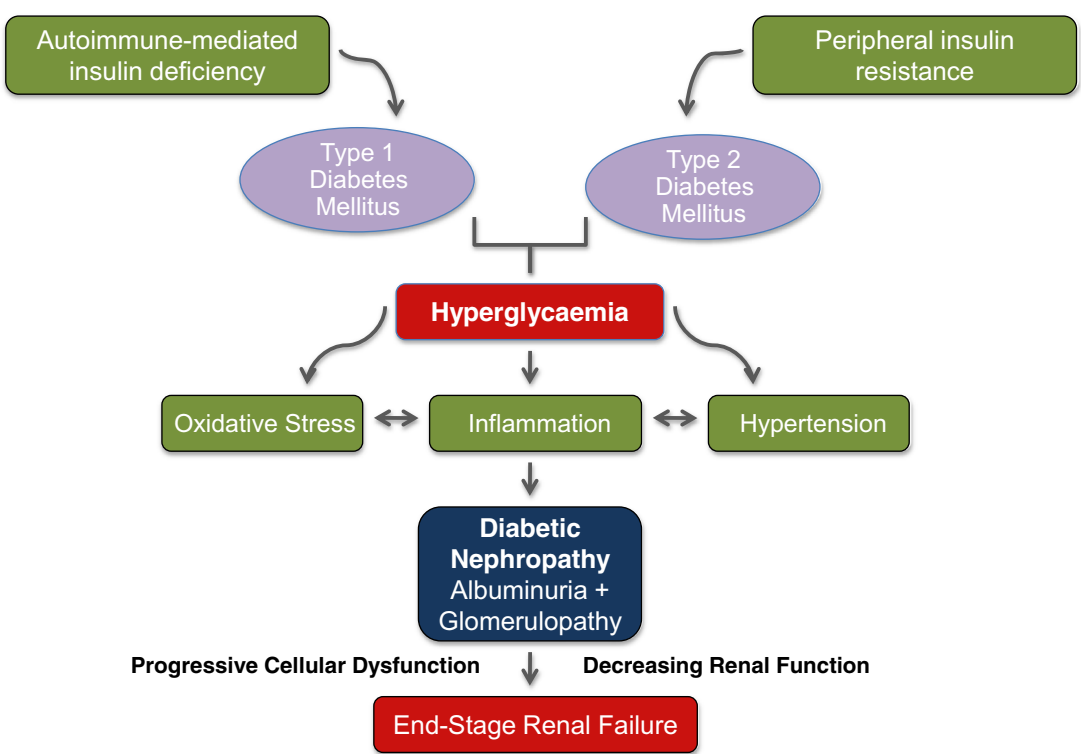


identified genes likely only account for a limited proportion of the observed phenotypic alterations in patients. Furthermore, it is possible that the pathogenic pathways differ between type 1 - and type 2-associated DN. Additionally, there is a distinct likelihood that unique mechanisms promote disease initiation versus disease progression.

Given that metabolic and haemodynamic changes are central pathogenic drivers of DN, approaches to target these mechanisms have become standards of care. However, it has become clear that the management of hypertension [20] and the pursuit of tight glycaemic control [21] are ineffective in stopping the inevitable renal deterioration in many patients. As a result, researchers are continuing to advance the understanding of DN, and as such, a wealth of novel therapeutic targets are being identified. Such developments will be essential to facilitate more effective diagnosis and treatment of DN.

\section{Diabetic Nephropathy: a Multi-cellular Affair}

By virtue of the complex anatomical structure and intricate physiology of normal kidneys, the intrinsic pathological features of DN involve a multitude of constituent renal cells. As highlighted previously, aberrant cellular signalling promotes characteristic histological changes within diabetic kidneys via the release of numerous secreted factors [22]. When attempting to rank dysfunction of specific cellular types, DN has often been viewed as a 'podocentric' disease, where the highly specialised podocytes are often described as the 'weakest link' among the renal cell population [22]. However, glomerular endothelial cells (GEnCs) and tubulointerstitial cells are increasingly implicated in the development and progression of DN [23-26].

A common feature of diabetic cardiovascular complications is diffuse endothelial injury, and $\mathrm{DN}$ is no exception. GEnCs are continuous, highly fenestrated cells that are covered by a thick glycoprotein layer of glycocalyx. It has been demonstrated that altered permeability of the GEnC layer can exacerbate levels of albuminuria, independent of ultrastructural changes in the podocytes [27]. Interestingly, in a study by Qi et al. which compared the transcriptomes of DN-resistant mice versus DNsusceptible mice, there was a significant downregulation of mitochondrial gene expression in the GEnCs of diabetic mice, which was associated with an increase in mitochondrial DNA lesions [24]. These results were confirmed using human tissue samples, where the authors correlated their findings with rapidly progressing DN [24]. These data provide new insights into the complexity of GEnC pathology in DN and align with the unifying concept that oxidative stress is central to diabetic vascular disease.

The myofibroblast represents another principal mediator of DN. These mesenchymal cells promote extracellular matrix accumulation and, hence, correlate positively with the amount of renal fibrosis. Whilst their role in disease progression has been firmly established, their origin remains controversial [28]. One explanation that has gained attention centres on the process of epithelial-to-mesenchymal transition (EMT). During EMT, renal epithelial cells undergo phenotypic changes, involving loss of epithelial markers and increased expression of mesenchymal genes [29]. Indeed, immunofluorescence has confirmed the presence of cells that stained positive for $\alpha$-smooth muscle actin ( $\alpha$-SMA), a smooth muscle differentiation marker expressed by myofibroblasts, in renal interstitial tissue originating from type 2 DM patients [30]. Such findings directly contrast with work published by Humphreys et al. who implemented Cre/Lox techniques to genetically label renal epithelial cells in a mouse model of renal fibrosis [31]. By using either $\beta$-galactosidase or red fluorescent protein as markers to track the fate of renal epithelial cells in vivo, Humpheys et al. found no evidence to support the interstitial migration of epithelial cells, highlighting a notable lack of $\alpha$ SMA-positive tubular cells [31]. This group suggested that the myofibroblast arises from the vascular pericyte and, hence, that therapeutic strategies against fibrotic kidney disease should target pericyte differentiation [31].

Recent papers from the Nieto and Kalluri groups have demonstrated a key role for the Snail transcription factor in kidney fibrosis and suggest that epithelial cells undergo a 'partial' EMT without continuing to the myofibroblast population [32, 33]. By the same token, the role of an analogous process called endothelial-to-mesenchymal transition (EndoMT) in DN is beginning to become clear. Evidence by $\mathrm{Li}$ et al. demonstrated the existence of this phenomenon in streptozotocin (STZ)-induced diabetic mice, a type 1 diabetic model [34], whilst a 2017 paper by Shang et al. extended this process to human samples by showing that nucleotide-binding oligomerization domain-containing protein 2 (NOD2) promotes EndoMT in DN biopsy tissue contributing to renal fibrosis [35]. Furthermore, recent publications have highlighted a novel role for bone marrow-derived myofibroblasts in renal fibrosis showing that pro-inflammatory macrophages are a source of collagen-producing myofibroblasts in both experimental and human models of DN [36, 37].

Consideration of any cell type in isolation is limited, as interactions between these cells are essential under both physiological and pathological conditions. In this way, there is a continuous bidirectional crosstalk that involves the exchange of numerous cytokines and growth factors. Moreover, the phenotypic and functional heterogeneities among renal cells, in particular renal endothelial cells, could imply the involvement of numerous unique signalling pathways. As such, it is now clear that the pathophysiology underpinning DN is a complex multi-molecular, multi-cellular process and therefore, targeting therapeutic strategies around more than one of these pathological targets may be a sensible approach. 


\section{Epigenetics: the Missing Piece of the Puzzle?}

For years, researchers have focused on the pivotal role of hyperglycaemia in the development of diabetic vascular complications. However, it is known that DN can develop even in those patients who show optimal blood glucose control [38, 39]. Moreover, the decline in renal function among DN patients is highly variable. In this regard, epigenetics is emerging as a next-generational paradigm to characterise DN. Epigenetics refers to the regulation of gene expression and phenotype that occurs without the need for DNA sequence changes. In keeping with the multi-factorial aetiology of $\mathrm{DM}$, it is clear that environmental factors and health behaviours influence disease pathology by inducing changes in epigenetically regulated mechanisms in chromatin, including DNA methylation and histone post-translational modifications [40].

DNA methylation involves a methyl group being added to the fifth carbon position of cytosine [41], and as a result, this process inversely regulates gene expression. DNA methylation is an important modification in the context of complex diseases. Altered DNA methylation profiles were detected in DN $[42,43]$, and such investigations have documented differential methylation of various mediators of glomerular cell apoptosis [41]. Furthermore, genome-wide methylation screening has identified genes that are selectively hypermethylated in fibrotic fibroblasts and thus, DNA methylation has been strongly implicated in fibroblast-induced renal fibrosis [44]. Bechtel et al. highlighted that DNA methylation changes regulate the actions of TGF- $\beta_{1}$ and that hypermethylation of RAS protein activator like 1 (RASAL1) correlated strongly with renal fibrogenesis [44].

Another compelling field of research is the role of noncoding RNAs (ncRNAs) in DN pathogenesis. Despite their appeal as novel biomarkers, the function of ncRNAs, such as microRNAs, is somewhat perplexing. This is evidenced by investigations into the role of mir-192, which demonstrated both pro- and anti-fibrotic capabilities [45, 46]. Also, it is important to realise that microRNAs rarely act in isolation. This fact was nicely highlighted by a report that TGF- $\beta_{1}$ indirectly regulated endoplasmic reticulum stress in diabetic mice, via targeting a mega-cluster of around 40 microRNAs [47]. The role of long non-coding RNAs (lncRNAs) in DN is now beginning to emerge. LncRNAs are $\geq 200$ nucleotides in length and function by means other than coding for proteins. As such, plasmacytoma variant translocation 1 (PVT1) was associated with diabetic ESRD in both type 1 and type 2 DM [48]. Further work highlighted that PVT1 expression was upregulated by hyperglycaemia and that knockdown of this IncRNA reduced the levels of key extracellular matrix proteins in human mesangial cells [49]. RNA sequencing analysis of renal glomeruli identified differential expression of the IncRNA, taurine upregulated 1 (Tug1) in the diabetic milieu
[50]. Recently, Li et al. showed that DN-associated upregulation of metastasis-associated lung adenocarcinoma transcript 1 (MALAT1) expression promoted renal tubular epithelial pyroptosis, an inflammatory form of programmed cell death, by amplifying the inflammatory response via modulation of ELAV-like RNA-binding protein 1 (EVAL1) signalling [51]. In the field of non-coding RNAs, it is expected that future work will uncover a plethora of targets associated with DN.

Histone deactylation is another component of epigenetic regulation, which converts permissive euchromatin to repressive heterochromatin and, hence, causes transcriptional silencing. Histone deacetylases (HDACs) have garnered recent attention due to their ability to effectively regulate the transcriptional network. Despite relatively few publications, it is likely that HDACs regulate key signalling events within the diabetic kidney. Evidence to support this hypothesis has been provided by both in vitro and in vivo research: For example, Noh et al. showed that HDAC-2 could promote extracellular matrix accumulation in both renal tubular epithelial cells and renal tissue of STZ-induced diabetic mice [52]. Increased expression of HDAC-2/4/5 was documented in renal tissues of diabetic animals and biopsy material from diabetic patients [53]. In particular, HDAC-4 was found to selectively exacerbate podocyte damage in $\mathrm{DN}$ [53].

Aside from histone modification, HDACs may regulate non-histone proteins. HDACs have thus been highlighted as potential regulators of pro-inflammatory gene expression in DM [54]. The ability of HDACs to interact with the innate and adaptive immune responses implicates these molecules in the inflammatory pathogenesis of the diabetic kidney. In particular, NLR family pyrin domain-containing 3 (NLRP3) and other inflammasome components act to trigger the inflammatory cascade in the diabetic kidney [55]. In keeping with this, activation of caspase- 1 has been shown to downregulate sirtuin 1 [56], a class III HDAC that usually engages in antioxidative and anti-inflammatory functions. In fact, sirtuin 1 downregulation leads to endothelial dysfunction in DM [57]. Altered HDAC function may therefore contribute to a wide range of pathological processes in DN. In contrast, HDAC-6 has been shown to negatively regulate NLRP3 activation [58] and thus, therapeutic modulation of HDACs may emerge as a useful therapeutic approach in DN. Although the precise roles of HDACs remain poorly understood, their ability to regulate both histone and non-histone proteins suggests that future research will unearth pivotal roles in DN pathology.

To move a step further, it is now becoming clear that these epigenetic capabilities confer 'memory' onto the cells. This phenomenon of 'metabolic memory' or more specifically 'hyperglycaemic memory' is common to both macro- and microvascular diabetic complications. In essence, this means that even transient periods of suboptimal glucose control can result in long-lasting consequences. This was evidenced in numerous models by the persistence of oxidative stress [59] 
and a failure to resolve glomerular basement membrane thickening [60] following blood glucose normalisation. It appears that these cells may become imprinted with epigenetic marks that are difficult, if not impossible, to reverse.

Even though the details of this complex process remain elusive, research has uncovered some important contributors. In this regard, it was suggested that prolonged epigenetic changes, as well as the long-term accumulation of advanced glycation end products, are compatible with this concept [61]. Furthermore, Geraldes et al. recently identified a novel marker of podocyte-specific memory [62]. Having previously demonstrated increased expression of the protein tyrosine phosphatase, SHP-1, in the renal cortex of type 1 diabetic mice, this group subsequently showed that this molecule remained elevated in podocytes even after systemic glucose normalisation [62]. When considering the vast amount of published data, there can be no doubt that the diabetic microenvironment induces profound epigenetic changes and, hence, alters the fine balance between beneficial and pathogenic transcriptome regulators. Furthermore, the ability to modulate metabolic memory may represent a powerful tool for the treatment of DN and other diseases. By exploring the relatively uncharted epigenetic landscape, researchers may uncover important insights into the molecular basis of DN.

\section{Current Standards of Care}

\section{Controlling Blood Glucose: the Holy Grail}

For years, effective glycaemic control has been hailed as the principal approach for reducing the incidence of diabetic complications. In the setting of DN, the benefits of tight glucose control were highlighted by examining the effects of sustained normoglycaemia following pancreatic transplantation. Fioretto et al. observed a reversal of renal lesions in DN patients after 10 years of normoglycaemia [63]. These landmark results suggested that precisely controlled glucose levels had the potential to promote significant reverse remodelling within the diabetic kidney. A number of authoritative studies have continued to support such benefits, including the Diabetes Control and Complications Trial (DCCT) [64]. In its type 1 DM cohort, one DCCT demonstrated that intensive glucose control improved various surrogate end points, including macroalbuminuria [64]. Additionally, the follow-up stage Epidemiology of Diabetes Interventions and Complications (EDIC) trial identified long-lasting risk reductions among the intensive therapy arm even when tight glucose control was no longer in place [65]. Further encouraging results came from the United Kingdom Prospective Diabetes Study (UKPDS) where intensive glycaemic control achieved an $11 \%$ reduction in glycosylated haemoglobin (HbA1c), a marker of glycaemic control in established DM, as well as a
$33 \%$ decrease in microalbuminuria levels when compared to conventional therapy at a median follow-up of 10 years [66]. Such results were replicated by the ADVANCE (Action in Diabetes and Vascular Disease: Preterax and Diamicron Modified Release Controlled Evaluation) trial, which observed a notable $65 \%$ risk reduction for ESRD in the intensively managed arm of the study [67]. These findings have farreaching ramifications, as reductions in renal disease indices should presumably associate with a reduced overall cardiovascular risk profile. However, whilst albuminuria is a wellestablished hallmark of DN, its sensitivity is somewhat limited. In this way, daily intra-patient fluctuations can confound its analysis. The ACCORD (Action to Control Cardiovascular Risk in Diabetes) trial also identified discordance between albuminuria and eGFR [68]. The ACCORD trial further raised concerns regarding strict glycaemic management by highlighting excessive mortality in the intensive therapy arm [68]. In a similar manner, analyses from the ADVANCE trial identified an association between hypoglycaemia and the development of adverse clinical effects [67]. These findings highlight the difficulties of pursuing an intensive regimen in diabetic patients. Considering the evidence supporting the central position of hyperglycaemia in the development of diabetic vascular complications, it seems likely that clinicians will continue to encourage their patients to aim for steady improvements in glycaemic control to reduce the risk of diabetic complications, such as DN.

\section{RAAS Inhibition: an Old Favourite}

The management of hypertension in DN is considered to be an important component in the clinical management of patients. Evidence for this was seen in the UKPDS trial where decreases in systolic blood pressure were associated with a decreased incidence of diabetic complications [66]. Inhibitors of the RAAS, including angiotensin-converting enzyme inhibitors (ACE-I) and angiotensin receptor blockers (ARB), are currently the first-line therapies for DN [69]. The positive effects of ARBs were highlighted by the RENAAL (Reduction of Endpoints in NIDDM with the Angiotensin II Antagonist Losartan) [70] and IRMA-2 (Irbesartan in Patients with Type 2 Diabetes and Microalbuminuria) [71] trials. Moreover, the DETAIL (Diabetics Exposed to Telmisartan and Enalapril) trial confirmed the consensus that both ACEIs and ARBs have equal efficacy in DN [72]. It is accepted that these agents provide benefits beyond mere blood pressure control, as evidenced by reductions in microalbuminuria [73]. By reducing intra-glomerular pressure, ACE-Is and ARBs have long-term effects on renal function even after cessation, suggesting that these anti-hypertensive drugs normalise some aspects of DN pathology [70]. Whilst these agents were initially used in all DN patients, recent guidelines recommend limiting their prescription to patients with 
proteinuria $>300 \mathrm{mg} /$ day [74]. Despite representing the current treatment of choice, these drugs merely slow DN progression. Additionally, traditional approaches to inhibit RAAS are limited by an increased risk of hyperkalaemia in patients with advanced disease. As such, there has been a concerted effort to optimise RAAS inhibition, whilst limiting conceivable adverse effects.

Recently, researchers questioned whether or not dual RAAS blockade could provide additional nephroprotective effects. In the ONTARGET (Ongoing Telmisartan Along and in Combination with Ramipril Global Endpoint Trial) study, combining the ARB, telmisartan, with the ACE-I, ramipril, did not improve proteinuria levels and, more importantly, was associated with increased rates of hyperkalaemia [75]. Similarly, the VA NEPHRON-D (Veterans Affairs Nephropathy in Diabetes) trial destroyed any hopes for dual blockade with an ACE-I and an ARB by confirming that this approach offered no significant benefit in the primary endpoints of renal disease progression or death [76]. In fact, the trial documented increased risks of serious adverse effects, including hyperkalaemia and acute kidney injury [76]. Despite considerable evidence that dual blockade should be avoided due to adverse side effects, there is an indication that an increasing proportion of patients is being managed in this way [77]. However, a recent glimmer of hope emerged following the development of novel potassium-binding drugs, including the oral non-absorbed polymer, patiromer. When used in combination with RAAS inhibition, only $15 \%$ of stage $3 / 4$ CKD patients developed hyperkalaemia compared to $60 \%$ among the placebo group [78]. Direct renin inhibition was considered as another approach to optimise RAAS modulation. Initial results from the AVOID trial were promising, as the addition of a direct renin inhibitor, aliskiren, to losartan therapy resulted in a $20 \%$ reduction in the urinary albumin to creatinine ratio [79]. However, in a similar manner to dual RAAS blockade, the larger ALTITUDE study was terminated after 2.7 years due to the absence of change in renal outcomes and excess adverse effect risk in the aliskiren arm of the trial [80]. As it stands, clinicians are currently limited to prescribing only one RAAS-targeting agent for DN patients. Taken together, it seems that conventional RAAS blockade is reaching its limits in terms of DN patient benefit.

\section{Novel Therapeutic Approaches}

\section{Anti-diabetic Drugs: Unanticipated Benefits}

Whilst researchers are often driven to develop new targets and novel therapies, an important part of medicine involves recognising when the solution is already within our grasp. As such, a number of currently available anti-diabetic drugs have shown promise in the treatment of DN. One particularly promising group is the sodium-glucose cotransporter-2 (SGLT2) inhibitors, such as empagliflozin. Beyond blood glucose control, these drugs possess potent renoprotective effects. A recent study by Wang et al. observed increased SGLT2 expression in renal biopsies from diabetic patients and that inhibition prevented $\mathrm{DN}$ development in $\mathrm{db} / \mathrm{db}$ mice, a leptin-deficient type 2 diabetic model [81]. Furthermore, the cardiovascular protection offered by these drugs has successfully translated into clinical studies, as noted by the EMPAREG OUTCOME trial [82]. Despite being prescribed for type 2 DM, SGLT2 inhibitors may afford further renoprotection to younger type $1 \mathrm{DM}$ patients, as demonstrated when empagliflozin was added to insulin therapy [83]. The ability of these drugs to target advanced kidney disease may, however, be questionable, as glucose-modulating effects lessened with decreasing eGFR. As such, the dedicated renal trial, CREDENCE [NCT02065791], should answer important questions regarding the potential of SGLT2 inhibitors to treat DN. Beyond SGLT2 inhibitors, clinical trials have suggested that glucagon-like peptide-1 (GLP-1) receptor agonists and dipeptidyl peptidase-4 (DPP-4) inhibitors can improve renal function, possibly independent of their anti-glycaemic properties $[84,85]$. These findings suggest that clinicians already have access to a wealth of established drugs that could potentially improve renal deterioration in DN patients.

\section{Beyond the Limits of RAAS Inhibition}

Under physiological conditions, two opposing arms of the RAAS remain in relative equilibrium: the pressor arm (ACE-I/angiotensin II) and the depressor arm (ACE-2/ Ang-(1-7)). There is increasing evidence that modulating the counter regulatory depressor arm can attenuate kidney damage. Chou et al. showed that hyperglycaemia lowered ACE-2 levels in rat proximal tubular cells [86]. Additionally, the positive effects of human recombinant ACE-2 in diabetic Akita mice were demonstrated by Oudit et al. with attenuated matrix expansion and blood pressure normalisation [87]. Moreover, whilst the Ang-(1-7) heptapeptide has been shown to alter various disease indices, it is evident that its short halflife will need to be improved to facilitate the development of clinically relevant formulations $[88,89]$. Despite the apparent ceiling in terms of the RAAS, new developments suggest that progress can still be made in improving the efficacy of targeting this system in DN.

\section{Oxidative Stress: Dampening the Destruction}

Oxidative stress has emerged as an important novel target for $\mathrm{DN}$, as imbalanced redox signalling resulting from the excessive generation of reactive oxygen species (ROS) exacerbates renal injury. Unfortunately, non-selective inhibition of oxidative stress has so far failed as a DN therapy, possibly due to 
unintentional interference with physiological ROS activity and the increasingly apparent complexity of redox signalling. More effective approaches may involve intensifying the effects of endogenous scavenging molecules, e.g. nuclear factor erythroid 2-like 2 (Nrf2) [90]. The potential to target Nrf2 was highlighted by Zheng et al. who showed that the Nrf2 agonists, sulphoraphane and cinnamic aldehyde, reduced oxidative stress and minimised pathological changes in the glomeruli of STZ-induced mice [91]. Clinical data from the BEAM (Bardoxolone Methyl Treatment: Renal Function in CKD/ Type 2 Diabetes) trial supported the efficacy of Nrf2 activation, since bardoxylone methyl was associated with improved renal function in patients with Type 2 DM [92]. The subsequent BEACON trial, however, was terminated prematurely due to an increased risk of cardiovascular events [93]. Another option to target this pathway may include the use of drugs with known and well-characterised safety profiles, such as the tetracycline antibiotic, minocycline, which stabilises Nrf2 and decreases oxidative stress [94].

Selective targeting of NADPH oxidase (NOX) enzymes, which are key modulators of the cellular redox state, may also hold therapeutic potential in DN. For example, Gorin et al. first showed that inhibiting NOX4, the most abundant renal isoform, with anti-sense oligonucleotides reduced glomerular expansion in diabetic rats [95]. Recent research also highlighted the ability of a dual NOX1/NOX4 inhibitor, GKT137831, to modulate renal pathology $[96,97]$. Specifically, Gorin et al. reported that GKT137831 suppressed diabetes-induced NOX activity and superoxide generation whilst reducing mesangial expansion in OVE26 mice [96]. More recently, Grey et al. demonstrated that delayed intervention with GKT137831 could effectively downregulate pro-inflammatory pathways in animal models with established DN [97]. Interestingly, additional atheroprotection was observed with low doses but was absent with higher concentrations of the drug. Therefore, it seems that the effects of GKT137831 are both dose- and tissue-dependent. Beyond this, a novel pan-NOX inhibitor, APX-115, was shown to have similar or superior efficacy to GKT137831 in DN [98]. This compound reduced both plasma creatinine levels and urinary albumin excretion in $\mathrm{db} / \mathrm{db}$ obese/diabetic mice [98]. Taken together, whilst these results appear promising, there remains a need to determine whether or not these NOX inhibitors can improve patient endpoints in large, randomised DN clinical trials.

\section{Inflammation: Calming the Storm}

In light of the intrinsic link between inflammation and hyperglycaemia, the ability to modulate key inflammatory cascades appears attractive for DN therapy. For instance, monocyte chemoattractant protein-1 (MCP-1) has emerged as a potential therapeutic target. MCP-1-mediated macrophage accumulation was subsequently linked to progressive functional deterioration [99]. Pre-clinical research has correspondingly highlighted the potential benefits of MCP-1 targeting, showing that it reduced diabetic glomerulosclerosis [100]. These positive effects were hinted at in a number of clinical studies, which showed that two agents targeting the MCP-1 axis, emapticap [101] and CCX140-B [102], decreased albuminuria levels in type $2 \mathrm{DM}$ patients.

Conflicting evidence has also emerged with regard to antiinflammatory agents. Despite the central role of TGF- $\beta$ in propagating inflammatory renal damage, clinical trials of TGF- $\beta$ inhibitors reported underwhelming results. As such, the promise of anti-TGF- $\beta_{1}$ therapy failed to materialise when a phase 2 study showed that there were no positive effects on DN progression [103]. The multi-functional capabilities of TGF- $\beta$ are increasingly being unveiled. In this way, research has challenged the previously well-accepted pathogenic role of TGF- $\beta$ by illustrating its potent anti-inflammatory [104] and anti-apoptotic potential [105]. Significantly, a recent paper published in JASN demonstrated that a dual specificity anti-TGF- $\beta$ antibody could reduce renal lesions when specifically targeted to the extracellular matrix of the kidney [106].

The T cell modulator, abatacept, which is currently licenced for rheumatoid arthritis, failed to preserve renal function in STZ-induced mice, as reflected by a failure to alter the albumin to creatinine ratio [107]. On the other hand, the potential to repurpose an old drug for DN has proved promising with regard to the methyxanthine derivative, pentoxifylline. The ability of this approved claudication therapy to inhibit TNF- $\alpha$ may have contributed to the promising results observed during the PREDIAN trial, where pentoxifylline caused significant reductions in urinary albumin excretion in DN patients [108]. Taken together, these studies suggest that specific elements of the immune system drive DN pathology and as such, translational efforts will need to focus toward their identification and targeting. It should also be noted that albuminuria may not be the most appropriate surrogate marker of efficacy for anti-inflammatory agents. As such, until novel biomarkers can be identified, future research will need to consider the evaluation of hard renal endpoints to monitor patient response to therapy.

\section{Renal Fibrosis: an Emerging Target}

Beyond inflammation and oxidative stress, many groups have proposed that targeting renal fibrosis may be an effective way to further slow functional deterioration in DN. The TGF- $\beta$ inhibitor, pirfenidone, has shown anti-fibrotic and renoprotective capabilities [109], but its use was associated with gastrointestinal side effects [110]. Interestingly, a phase I trial of anti-connective tissue growth factor (CTGF) monoclonal antibodies demonstrated an ability to decrease the urinary albumin to creatinine ratio [111] but following the termination of a phase II trial, it seems that this strategy is no longer being 
pursued. Of interest, the DPP-4 inhibitor, linagliptin, demonstrated notable anti-fibrotic effects via the inhibition of TGF- $\beta 2$-induced EndoMT in a type 1 diabetic mouse model [112]. Reductions in the levels of gremlin 1 (GREM1) have also been shown to attenuate diabetic and other forms of renal fibrosis [113-115]. Other options to target renal fibrosis have recently included targeting homeodomain-interacting protein kinase 2 (HIPK2) [116] or SMAD7 gene transfer [117]. MicroRNA modulation has shown significant promise, as the inhibition of mir-192 successfully repressed renal fibrosis in DN [118]. Mir-103b inhibition also improved renal tubulointerstitial fibrosis by suppressing Snail-induced EMT [119]. Vitamin D deficiency has been linked to an increased severity of structural renal alterations [120]. In a study by Tian et al., administration of active vitamin $\mathrm{D}$ attenuated renal fibrosis and protected renal function [121], making this approach a potential future consideration in the management of DN patients. Many of these therapies remain in the pre-clinical phase, and their efficacy in human patients has yet to be demonstrated.

\section{Harnessing Cellular Defences}

An alternative approach to $\mathrm{DN}$ treatment could revolve around the use of agents to harness the body's cytoprotective pathways. Thus, instead of blocking disease-driving molecules, it may be more fruitful to focus on those agents that can mobilise our innate molecular defences. Such approaches may be able to circumvent the damaging effects of glucotoxicity. One relevant mechanism is the nutrient-sensitive process of autophagy, which is downregulated in DN [122]. Autophagy is a tightly regulated lysosomal process that results in the degradation of unwanted cytoplasmic constituents [123] and is thus responsible for maintaining intra-cellular homeostasis. Moreover, autophagy is activated in response to cellular stresses, including nutrient deprivation, oxidative stress and metabolic abnormalities. Evidence suggests that in DN, autophagic systems become insufficient and that deficient autophagy has the potential to accelerate renal fibrosis [124]. Importantly, key features of DN were recapitulated in animal models via enhanced activation of the autophagy-inhibiting gene, mechanistic target of rapamycin kinase (mTOR) [125]. Moreover, levels of the mitochondrial protector, sirtuin 1 , were significantly reduced in the diabetic kidney [126].

Considering the proposed importance of autophagy in the maintenance of renal homeostasis, the complexities of this mechanism remain incompletely understood. Whilst this process is impaired in both constituent cells of the glomeruli and the renal tubules, its impact on podocyte destruction is particularly interesting. Podocytes are terminally differentiated epithelial cells, which form an important layer in the molecular sieve of the glomerular basement membrane. Their role in DN pathogenesis was confirmed in a landmark publication by Pagtalunan et al., which showed that a reduction in podocyte number was positively associated with albuminuria [127]. Given their inability to repair following damage, these cells demonstrate high basal autophagic activity under physiological conditions. A more recent study reported that podocytespecific autophagy-deficient diabetic mice developed significant proteinuria, whilst also confirming the lack of autophagy in renal biopsy tissue, which displayed intra-cellular accumulation of p62, a specific autophagy target [128]. Furthermore, another group demonstrated that hyperglycaemia-induced overexpression of the permeability-inducing molecule vascular endothelial growth factor (VEGF) could be enhanced by the autophagy inhibitor, 3-methyladenine [129]. These findings were in line with previous research demonstrating that this autophagy-driven phenomenon was sensitive to changing levels of ROS [129]. However, caution must be exercised when interpreting and undertaking autophagy-focused studies so as to avoid the extrapolation of findings regarding the amount of autophagy-produced substrates to variations in autophagy instability among cells.

As such, the ability to modulate autophagy may provide an exciting mechanism to reverse DN pathology. Indeed, calorie restriction in diabetic rats proved to regulate disordered autophagy by upregulating sirtuin 1 [130] and by suppressing mTOR [131]. Given the widely accepted health-prolonging benefits of calorie restriction, the adherence of patients to intense dietary modifications may be a useful additive approach. However, the degree of calorie restriction remains poorly defined and implementation of such radical health behaviours may ultimately be difficult to implement. Alternatively, using compounds to modulate these nutrient-sensitive pathways may be a more feasible strategy. Such agents could include the anti-diabetic drug metformin [132] or the herbal compound paeoniflorin [133]. In particular, modulating the expression of AMP-activated protein kinase (AMPK), an autophagy activator and target of metformin, has proven worthwhile. Kim et al. found that phosphorylation of AMPK by resveratrol prevented renal lipotoxicity and glucotoxicity [134]. In addition, Lee et al. demonstrated that pharmacological activation of AMPK successfully attenuated renal hypertrophy in diabetic rats [135]. Moreover, the macrolide compound, rapamycin, has proven capable of inhibiting the mTORC1 pathway and its potent ability to modulate DN pathology was demonstrated in various animal models [136, 137]. To date, the potential targeting of this intricate cellular defence mechanism appears promising. However, this therapeutic strategy is still in its infancy and as such, further research is required to inform more sophisticated approaches to reactivate autophagy.

\section{Stem Cells: the Emerging Frontier}

Recent years have seen increasing attempts to move the field of stem cell biology into the clinical environment. However, 
the strategies taken to harness the therapeutic potential of stem cells have proven to be challenging. Whilst the clinical use of embryonic stem cells is hampered by a string of ethical concerns, the emergence of induced pluripotent stem cells (iPSCs) has provided an attractive alternative. A number of groups have reported the generation of renal subtypes from iPSCs [138-140]. For example, Lam et al. produced cells characteristic of intermediate mesoderm, which subsequently expressed characteristic proximal tubular markers [140]. It should, however, be pointed out that iPSC-derived populations display significant phenotypic variability. Beyond the difficulties of generating pure cell populations, methods for renal lineage differentiation require additional optimisation in order to produce the high-quality cell yield needed for therapeutic purposes.

Considering that the kidney is essentially a mesodermal organ, focus has understandably shifted to mesenchymal stem cells (MSCs), which are multi-potent bone marrow-derived cells and connective tissue progenitors. Interestingly, Wang et al. showed that intra-arterial administration of MSCs to type 1 diabetic Sprague Dawley rats reduced podocyte effacement and prevented the loss of glomerular nephrin and podocin [141]. Whilst this treatment did not alter blood glucose levels, Ezquer et al. reported that MSC-treated diabetic mice demonstrated improved glycaemic levels within 1 week and that remarkably, euglycaemia was achieved within 1 month posttreatment [142].

As well as their regenerative ability, MSCs possess potent immunomodulatory functions and are capable of secreting various signalling molecules by which, among other actions, they are able to antagonise TGF- $\beta$ signalling [143]. Indeed, these cells are known to exert many of their effects through paracrine signalling. Moreover, exosomes contained in MSCconditioned media were recently shown to be key orchestrators of their therapeutic actions [144]. Taken together, it is evident that MSCs hold significant therapeutic potential; the ongoing EU-funded NEPHSTROM trial [NCT02585622] will provide essential safety information for allogenic bone marrow-derived MSC treatment in DN patients.

\section{Conclusions and Perspectives}

DN is a serious complication that develops in approximately one third of diabetic patients. Unfortunately, despite the obvious benefits derived from managing hyperglycaemia and haemodynamic dysregulation, many patients progress to ESRD requiring dialysis or transplant. Although there are continued advancements in the field, the underlying molecular mechanisms of DN have yet to be completely elucidated. Even

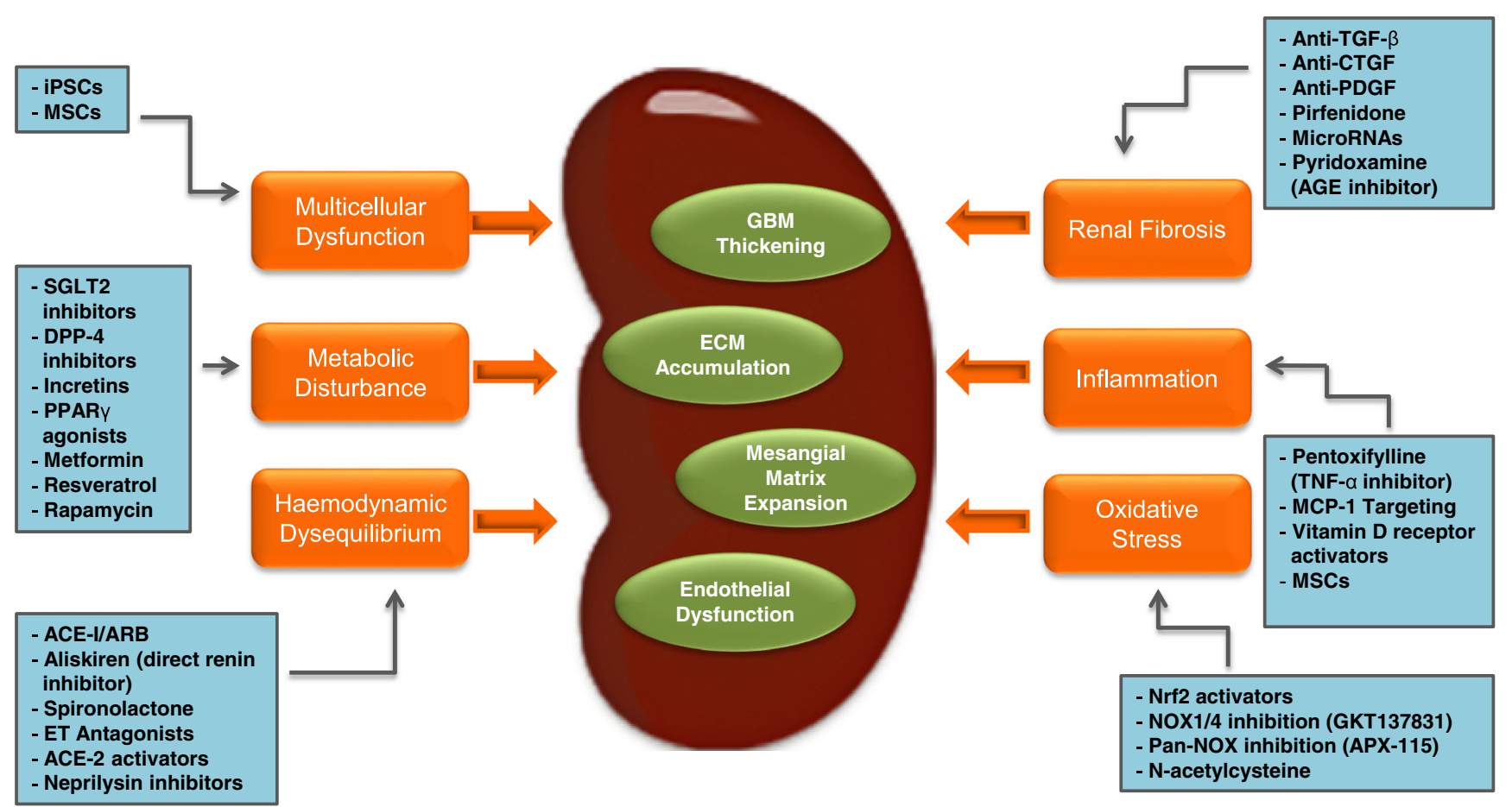

Fig. 2 Potential target pathways and therapeutic agents currently under investigation for the treatment of diabetic nephropathy: iPSCs induced pluripotent stem cells, MSCs mesenchymal stem cells, SGLT2 sodiumglucose cotransporter-2, DPP-4 dipeptidyl peptidase-4, PPAR $\gamma$ peroxisome proliferator-activated receptor gamma, ACE-I/ARB angiotensin-converting enzyme inhibitor, angiotensin receptor blocker,
ET endothelin receptor, ACE-2 angiotensin-converting enzyme 2, GBM glomerular basement membrane, ECM extracellular matrix, TGF- $\beta$ tissue growth factor-beta, CTGF connective tissue growth factor, PDGF platelet-derived growth factor, AGE advanced glycation end products, MCP-1 monocyte chemoattractant protein-1, Nrf2 nuclear factor erythroid 2-like 2 
though hyperglycaemia-induced TGF- $\beta$ upregulation has emerged as a central pathogenic mechanism in DN, research has highlighted the contributions of additional pleiotropic cellular events. These changes are set against the background of a multi-layered cellular communication system within the kidney. It has therefore been proposed that DN cannot be considered as a simple 'microvascular' disease. Future efforts to refine our understanding of DN pathophysiology will be necessary to resolve the current debates, determine the relative importance of newly identified pathogenic mechanisms and prioritise novel therapeutic targets.

Whilst the complex nature of DN is likely responsible for many of the obstacles that are preventing the successful translation of novel targets into effective DN therapeutics, this complexity has provided researchers with a wealth of pathways available for potential targeting (Fig. 2). Such research should aid in the translation of new DN-targeted therapies that will improve patient outcomes. Oxidative stress, inflammation and fibrosis are pivotal interlocking pathogenic processes that contribute to DN. As such, the ability to target each of these deleterious mechanisms presents an exciting therapeutic avenue. Despite appearing as obvious targets, modulation of these pathways has not been straightforward. Novel approaches, including cellular therapies or the modulation of autophagy, may eventually revolutionise DN treatment. Nevertheless, many challenges remain for researchers in the field of DN. These range from the use of unrepresentative animal models to poorly defined clinical trial inclusion criteria, which make it almost impossible to confirm causality. Additionally, the ability to measure traditional renal endpoints necessitates the use of resource-intensive, long-lasting clinical trials. In this regard, multi-level 'omics' studies may help to identify appropriate DN biomarkers. Improved design and execution of clinical trials for DN are urgently needed. Drug safety is another major concern, as the suboptimal compensatory mechanisms in DN patients make them particularly vulnerable to adverse drug effects. Moreover, the need to foresee potential drug interactions will be an important step to improving patient outcomes.

Further to cutting-edge developments in genomic technology, research has pinpointed several key pathways related to DN initiation and progression. The true challenge, however, will be to overcome the inherent heterogeneity of DN to identify novel drug targets that are relevant to large patient numbers. In essence, the successful management of DN will hinge on the implementation of a multi-disciplinary, multi-target approach, in which novel therapeutics are coupled with the traditional management strategies of promoting glucose control, blood pressure management and continued engagement in allround positive health behaviours. Such complexity dictates that one treatment for DN will not cure all, so more personalised approaches will be required. In essence, it is only with this realisation in mind that medical professionals will be best equipped to manage the DN patient population.
Continued investment in basic and clinical researches will be essential to successfully translate exciting pre-clinical data into reliable biomarkers and effective therapeutics for DN. Such work holds the potential to bring about measurable changes in clinical outcomes and to establish an improved quality and quantity of life for the DN patient population.

Acknowledgements The authors' work is supported by the British Heart Foundation, the Health Research Board of Ireland, Diabetes UK and The Wellcome Trust.

Open Access This article is distributed under the terms of the Creative Commons Attribution 4.0 International License (http:// creativecommons.org/licenses/by/4.0/), which permits unrestricted use, distribution, and reproduction in any medium, provided you give appropriate credit to the original author(s) and the source, provide a link to the Creative Commons license, and indicate if changes were made.

\section{References}

1. American Diabetes Association. Diagnosis and classification of diabetes mellitus. Diabetes Care. 2014;37:S81-90.

2. Forbes JM, Cooper ME. Mechanisms of diabetic complications. Physiol Rev. 2013;93:137-88.

3. Toth-Manikowski S, Atta MG. Diabetic kidney disease: pathophysiology and therapeutic targets. J Diabetes Res. 2015;2015: 697010.

4. Saran R, Li Y, Robinson B, et al. US renal data system 2014 annual data report: epidemiology of kidney disease in the United States. Am J Kidney Dis. 2015;66:S1-305.

5. Checherita IA, Turcu F, Dragomirescu RF, Ciocalteu A. Chronic complications in hemodialysis: correlations with primary renal disease. Romanian J Morphol Embryol. 2010;51:21-6.

6. Stoumpos S, Jardine AG, Mark PB. Cardiovascular morbidity and mortality after kidney transplantation. Transpl Int. 2015;28:10-21.

7. Groop PH, Thomas MC, Moran JL, et al. The presence and severity of chronic kidney disease predicts all-cause mortality in type 1 diabetes. Diabetes. 2009;58:1651-8.

8. Afkarian M, Sachs MC, Kestenbaum B, et al. Kidney disease and increased mortality risk in type 2 diabetes. J Am Soc Nephrol. 2013;24:302-8.

9. Cooper ME. Interaction of metabolic and haemodynamic factors in mediating experimental diabetic nephropathy. Diabetologia. 2001;44:1957-72.

10. Schena FP, Gesualdo L. Pathogenetic mechanisms of diabetic nephropathy. J Am Soc Nephrol. 2005;16:S30-3.

11. Yamamoto T, Nakamura T, Noble NA, Ruoslahti E, Border WA. Expression of transforming growth factor beta is elevated in human and experimental diabetic nephropathy. Proc Natl Acad Sci U S A. 1993;90:1814-8.

12. Wahab NA, Schaefer L, Weston BS, et al. Glomerular expression of thrombospondin-1, transforming growth factor beta and connective tissue growth factor at different stages of diabetic nephropathy and their interdependent roles in mesangial response to diabetic stimuli. Diabetologia. 2005;48:2650-60.

13. Sharma K, Ziyadeh FN. Renal hypertrophy is associated with upregulation of TGF-beta 1 gene expression in diabetic BB rat and NOD mouse. Am J Phys. 1994;267:F1094-101.

14. Pantsulaia T. Role of TGF-beta in pathogenesis of diabetic nephropathy. Georgian Med News. 2006;131:13-8.

15. Derynck R, Zhang YE. Smad-dependent and Smad-independent pathways in TGF-beta family signalling. Nature. 2003;425:577-84. 
16. Kim BK, Moon KC, Hong HK, Lee HS. Activation of the TGFbeta/Smad signaling pathway in focal segmental glomerulosclerosis. Kidney Int. 2003;64:1715-21.

17. Sato M, Muragaki Y, Saika S, Roberts AB, Ooshima A. Targeted disruption of TGF-beta1/Smad3 signaling protects against renal tubulointerstitial fibrosis induced by unilateral ureteral obstruction. J Clin Invest. 2003;112:1486-94.

18. Yan Y, Ma L, Zhou X, et al. Src inhibition blocks renal interstitial fibroblast activation and ameliorates renal fibrosis. Kidney Int. 2016;89:68-81.

19. Sandholm N, Salem RM, McKnight AJ, et al. New susceptibility loci associated with kidney disease in type 1 diabetes. PLoS Genet. 2012;8:e1002921.

20. Sjolie AK, Chaturvedi N, Fuller J. Effect of lisinopril on progression of retinopathy and microalbuminuria in normotensive subjects with insulin-dependent diabetes mellitus. Ugeskr Laeger. 1999;161:949-52.

21. Yadav S. Glycaemic control in diabetic kidney disease patients. Clin Queries: Nephrol. 2012;1:111-4.

22. Maezawa Y, Takemoto M, Yokote K. Cell biology of diabetic nephropathy: roles of endothelial cells, tubulointerstitial cells and podocytes. J Diabetes Investig. 2015;6:3-15.

23. Satchell SC, Tooke JE. What is the mechanism of microalbuminuria in diabetes: a role for the glomerular endothelium? Diabetologia. 2008;51:714-25.

24. Qi H, Casalena G, Shi S, et al. Glomerular endothelial mitochondrial dysfunction is essential and characteristic of diabetic kidney disease susceptibility. Diabetes. 2017;66:763-78.

25. Tonolo G, Cherchi S. Tubulointerstitial disease in diabetic nephropathy. Int J Nephrol Renovasc Dis. 2014;7:107-15.

26. Tang SC, Lai KN. The pathogenic role of the renal proximal tubular cell in diabetic nephropathy. Nephrol Dial Transplant. 2012;27:3049-56.

27. Davis B, Dei Cas A, Long DA, et al. Podocyte-specific expression of angiopoietin-2 causes proteinuria and apoptosis of glomerular endothelia. J Am Soc Nephrol. 2007;18:2320-9.

28. Loeffler I, Wolf G. Epithelial-to-mesenchymal transition in diabetic nephropathy: fact or fiction? Cell. 2015;4:631-52.

29. Liu Y. Epithelial to mesenchymal transition in renal fibrogenesis: pathologic significance, molecular mechanism, and therapeutic intervention. J Am Soc Nephrol. 2004;15:1-12.

30. Mandache E, Gherghiceanu M, Serafinceanu C, Penescu M, Mircescu G. Myofibroblast involvement in tubular basement membrane remodeling in type II diabetic nephropathy. Romanian J Morphol Embryol. 2011;52:75-9.

31. Humphreys BD, Lin SL, Kobayashi A, et al. Fate tracing reveals the pericyte and not epithelial origin of myofibroblasts in kidney fibrosis. Am J Pathol. 2010;176:85-97.

32. Lovisa S, LeBleu VS, Tampe B, et al. Epithelial-to-mesenchymal transition induces cell cycle arrest and parenchymal damage in renal fibrosis. Nat Med. 2015;21:998-1009.

33. Grande MT, Sanchez-Laorden B, Lopez-Blau C, et al. Snail1Induced partial epithelial-to-mesenchymal transition drives renal fibrosis in mice and can be targeted to reverse established disease. Nat Med. 2015;21:989-97.

34. Li J, Qu X, Bertram JF. Endothelial-myofibroblast transition contributes to the early development of diabetic renal interstitial fibrosis in streptozotocin-induced diabetic mice. Am J Pathol. 2009;175:1380-8.

35. Shang J, Zhang Y, Jiang Y, et al. NOD2 Promotes endothelial-tomesenchymal transition of glomerular endothelial cells via MEK/ ERK signaling pathway in diabetic nephropathy. Biochem Biophys Res Commun. 2017;484:435-41.

36. Meng XM, Wang S, Huang XR, et al. Inflammatory macrophages can transdifferentiate into myofibroblasts during renal fibrosis. Cell Death Dis. 2016;7:e2495.
37. Wang S, Meng XM, Ng YY, et al. TGF-beta/Smad3 signalling regulates the transition of bone marrow-derived macrophages into myofibroblasts during tissue fibrosis. Oncotarget. 2016;7:8809-22.

38. Filippone EJ, Gupta A, Farber JL. Normoglycemic diabetic nephropathy: the role of insulin resistance. Case Rep Nephrol Urol. 2014;4:137-43.

39. Bakris GL. Recognition, pathogenesis, and treatment of different stages of nephropathy in patients with type 2 diabetes mellitus. Mayo Clin Proc. 2011;86:444-56.

40. Reddy MA, Tak Park J, Natarajan R. Epigenetic modifications in the pathogenesis of diabetic nephropathy. Semin Nephrol. 2013;33:341-53.

41. Bell CG, Teschendorff AE, Rakyan VK, Maxwell AP, Beck S, Savage DA. Genome-wide DNA methylation analysis for diabetic nephropathy in type 1 diabetes mellitus. BMC Med Genet. 2010;3:33

42. Smyth LJ, McKay GJ, Maxwell AP, McKnight AJ. DNA hypermethylation and DNA hypomethylation is present at different loci in chronic kidney disease. Epigenetics. 2014;9:366-76.

43. Wing MR, Devaney JM, Joffe MM, et al. DNA methylation profile associated with rapid decline in kidney function: findings from the CRIC study. Nephrol Dial Transplant. 2014;29:864-72.

44. Bechtel W, McGoohan S, Zeisberg EM, et al. Methylation determines fibroblast activation and fibrogenesis in the kidney. Nat Med. 2010;16:544-50.

45. Kato M, Zhang J, Wang M, et al. MicroRNA-192 in diabetic kidney glomeruli and its function in TGF-beta-induced collagen expression via inhibition of E-box repressors. Proc Natl Acad Sci U S A. 2007;104:3432-7.

46. Krupa A, Jenkins R, Luo DD, Lewis A, Phillips A, Fraser D. Loss of MicroRNA-192 promotes fibrogenesis in diabetic nephropathy. J Am Soc Nephrol. 2010;21:438-47.

47. Kato M, Wang M, Chen Z, et al. An endoplasmic reticulum stressregulated lncRNA hosting a microRNA megacluster induces early features of diabetic nephropathy. Nat Commun. 2016;7:12864.

48. Hanson RL, Craig DW, Millis MP, et al. Identification of PVT1 as a candidate gene for end-stage renal disease in type 2 diabetes using a pooling-based genome-wide single nucleotide polymorphism association study. Diabetes. 2007;56:975-83.

49. Alvarez ML, DiStefano JK. Functional characterization of the plasmacytoma variant translocation 1 gene (PVT1) in diabetic nephropathy. PLoS One. 2011;6:e18671.

50. Long J, Badal SS, Ye Z, et al. Long noncoding RNA Tug1 regulates mitochondrial bioenergetics in diabetic nephropathy. J Clin Invest. 2016;126:4205-18.

51. Li X, Zeng L, Cao C, et al. Long noncoding RNA MALAT1 regulates renal tubular epithelial pyroptosis by modulated miR$23 \mathrm{c}$ targeting of ELAVL1 in diabetic nephropathy. Exp Cell Res. 2017;350:327-35.

52. Noh H, Oh EY, Seo JY, et al. Histone deacetylase-2 is a key regulator of diabetes- and transforming growth factor-beta1induced renal injury. Am J Physiol Renal Physiol. 2009;297: F729-39.

53. Wang X, Liu J, Zhen J, et al. Histone deacetylase 4 selectively contributes to podocyte injury in diabetic nephropathy. Kidney Int. 2014;86:712-25.

54. Christensen DP, Dahllof M, Lundh M, et al. Histone deacetylase (HDAC) inhibition as a novel treatment for diabetes mellitus. Mol Med. 2011;17:378-90.

55. Qiu YY, Tang LQ. Roles of the NLRP3 inflammasome in the pathogenesis of diabetic nephropathy. Pharmacol Res. 2016;114: 251-64.

56. Chalkiadaki A, Guarente L. High-fat diet triggers inflammationinduced cleavage of SIRT1 in adipose tissue to promote metabolic dysfunction. Cell Metab. 2012;16:180-8. 
57. Li Q, Kim YR, Vikram A, et al. P66Shc-induced microRNA-34a causes diabetic endothelial dysfunction by downregulating sirtuin1. Arterioscler Thromb Vasc Biol. 2016;36:2394-403.

58. Hwang I, Lee E, Jeon SA, Yu JW. Histone deacetylase 6 negatively regulates NLRP3 inflammasome activation. Biochem Biophys Res Commun. 2015;467:973-8.

59. Kowluru RA, Abbas SN, Odenbach S. Reversal of hyperglycemia and diabetic nephropathy: effect of reinstitution of good metabolic control on oxidative stress in the kidney of diabetic rats. J Diabetes Complicat. 2004;18:282-8.

60. Gotzsche O, Gundersen HJ, Osterby R. Irreversibility of glomerular basement membrane accumulation despite reversibility of renal hypertrophy with islet transplantation in early experimental diabetes. Diabetes. 1981;30:481-5.

61. Yamagishi S, Matsui T. Advanced glycation end products, oxidative stress and diabetic nephropathy. Oxidative Med Cell Longev. 2010;3:101-8.

62. Lizotte F, Denhez B, Guay A, Gevry N, Cote AM, Geraldes P. Persistent insulin resistance in podocytes caused by epigenetic changes of SHP-1 in diabetes. Diabetes. 2016;65:3705-17.

63. Fioretto P, Steffes MW, Sutherland DE, Goetz FC, Mauer M. Reversal of lesions of diabetic nephropathy after pancreas transplantation. N Engl J Med. 1998;339:69-75.

64. Nathan DM, Genuth S, Lachin J, et al. The diabetes control and complications trial research group. The effect of intensive treatment of diabetes on the development and progression of long-term complications in insulin-dependent diabetes mellitus. N Engl J Med. 1993;329:977-86.

65. de Boer IH, DCCT/EDIC Research Group. Kidney disease and related findings in the diabetes control and complications trial/ epidemiology of diabetes interventions and complications study. Diabetes Care. 2014;37:24-30.

66. UK Prospective Diabetes Study (UKPDS) Group. Intensive blood-glucose control with sulphonylureas or insulin compared with conventional treatment and risk of complications in patients with type 2 diabetes (UKPDS 33). Lancet. 1998;352:837-53.

67. Perkovic V, Heerspink HL, Chalmers J, et al. Intensive glucose control improves kidney outcomes in patients with type 2 diabetes. Kidney Int. 2013;83:517-23.

68. Action to Control Cardiovascular Risk in Diabetes Study Group, Gerstein HC, Miller ME, et al. Effects of intensive glucose lowering in type 2 diabetes. N Engl J Med. 2008;358:2545-59.

69. Quiroga B, Arroyo D, de Arriba G. Present and future in the treatment of diabetic kidney disease. J Diabetes Res. 2015;2015: 801348

70. Bakris GL, Weir MR, Shanifar S, et al. Effects of blood pressure level on progression of diabetic nephropathy: results from the RENAAL study. Arch Intern Med. 2003;163:1555-65.

71. Parving HH, Lehnert $\mathrm{H}$, Brochner-Mortensen J, et al. The effect of irbesartan on the development of diabetic nephropathy in patients with type 2 diabetes. N Engl J Med. 2001;345:870-8.

72. Barnett AH, Bain SC, Bouter P, et al. Angiotensin-receptor blockade versus converting-enzyme inhibition in type 2 diabetes and nephropathy. N Engl J Med. 2004;351:1952-61.

73. Viberti G, Wheeldon NM, MicroAlbuminuria Reduction With VALsartan (MARVAL) Study Investigators. Microalbuminuria reduction with valsartan in patients with type 2 diabetes mellitus: a blood pressure-independent effect. Circulation. 2002;106:672-8.

74. Taler SJ, Agarwal R, Bakris GL, et al. KDOQI US commentary on the 2012 KDIGO clinical practice guideline for management of blood pressure in CKD. Am J Kidney Dis. 2013;62:201-13.

75. Mann JF, Schmieder RE, McQueen M, et al. Renal outcomes with telmisartan, ramipril, or both, in people at high vascular risk (the ONTARGET study): a multicentre, randomised, double-blind, controlled trial. Lancet. 2008;372:547-53.
76. Fried LF, Emanuele N, Zhang JH, et al. Combined angiotensin inhibition for the treatment of diabetic nephropathy. N Engl J Med. 2013;369:1892-903.

77. Tylicki L, Jakubowska A, Lizakowski S, Swietlik D, Rutkowski B. Management of renin-angiotensin system blockade in patients with chronic kidney disease under specialist care. Retrospective cross-sectional study. J Renin-Angiotensin-Aldosterone Syst. 2015;16:145-52.

78. Bakris GL, Pitt B, Weir MR, et al. Effect of patiromer on serum potassium level in patients with hyperkalemia and diabetic kidney disease: the AMETHYST-DN randomized clinical trial. JAMA. 2015;314:151-61.

79. Parving HH, Persson F, Lewis JB, Lewis EJ, Hollenberg NK, Study Investigators AVOID. Aliskiren combined with losartan in type 2 diabetes and nephropathy. N Engl J Med. 2008;358:2433-46.

80. Parving HH, Brenner BM, McMurray JJ, et al. Cardiorenal end points in a trial of aliskiren for type 2 diabetes. $\mathrm{N}$ Engl $\mathrm{J}$ Med. 2012;367:2204-13.

81. Wang XX, Levi J, Luo Y, et al. SGLT2 Expression is increased in human diabetic nephropathy: SGLT2 inhibition decreases renal lipid accumulation, inflammation and the development of nephropathy in diabetic mice. J Biol Chem. 2017;292:5335-48.

82. Zinman B, Lachin JM, Inzucchi SE, et al. Empagliflozin, cardiovascular outcomes, and mortality in type 2 diabetes. $\mathrm{N}$ Engl $\mathrm{J}$ Med. 2015;373:2117-28.

83. Cherney DZ, Perkins BA, Soleymanlou N, et al. Renal hemodynamic effect of sodium-glucose cotransporter 2 inhibition in patients with type 1 diabetes mellitus. Circulation. 2014;129:587-97.

84. Hattori S. Sitagliptin reduces albuminuria in patients with type 2 diabetes. Endocr J. 2011;58:69-73.

85. Sakata K, Hayakawa M, Yano Y, et al. Efficacy of alogliptin, a dipeptidyl peptidase-4 inhibitor, on glucose parameters, the activity of the advanced glycation end product (AGE) - receptor for AGE (RAGE) axis and albuminuria in Japanese type 2 diabetes. Diabetes Metab Res Rev. 2013;29:624-30.

86. Chou CH, Chuang LY, Lu CY, Guh JY. Interaction between TGFbeta and ACE2-Ang-(1-7)-mas pathway in high glucose-cultured NRK-52E cells. Mol Cell Endocrinol. 2013;366:21-30.

87. Oudit GY, Liu GC, Zhong J, et al. Human recombinant ACE2 reduces the progression of diabetic nephropathy. Diabetes. 2010;59:529-38.

88. Padda RS, Shi Y, Lo CS, Zhang SL, Chan JS. Angiotensin-(1-7): a novel peptide to treat hypertension and nephropathy in diabetes? J Diabetes Metab 2015;6:615.

89. Shi Y, Lo CS, Padda R, et al. Angiotensin-(1-7) prevents systemic hypertension, attenuates oxidative stress and tubulointerstitial fibrosis, and normalizes renal angiotensin-converting enzyme 2 and mas receptor expression in diabetic mice. Clin Sci (Lond). 2015;128:649-63.

90. Arellano-Buendia AS, Tostado-Gonzalez M, Garcia-Arroyo FE, et al. Anti-inflammatory therapy modulates Nrf2-Keap1 in kidney from rats with diabetes. Oxidative Med Cell Longev. 2016;2016: 4693801.

91. Zheng H, Whitman SA, Wu W, et al. Therapeutic potential of Nrf2 activators in streptozotocin-induced diabetic nephropathy. Diabetes. 2011;60:3055-66.

92. Pergola PE, Raskin P, Toto RD, et al. Bardoxolone methyl and kidney function in CKD with type 2 diabetes. $\mathrm{N}$ Engl $\mathrm{J}$ Med. 2011;365:327-36.

93. de Zeeuw D, Akizawa T, Audhya P, et al. Bardoxolone methyl in type 2 diabetes and stage 4 chronic kidney disease. N Engl J Med. 2013;369:2492-503.

94. Shahzad K, Bock F, Al-Dabet MM, et al. Stabilization of endogenous Nrf2 by minocycline protects against Nlrp3-inflammasome induced diabetic nephropathy. Sci Rep. 2016;6:34228. 
95. Gorin Y, Block K, Hernandez J, et al. Nox4 NAD(P)H oxidase mediates hypertrophy and fibronectin expression in the diabetic kidney. J Biol Chem. 2005;280:39616-26.

96. Gorin Y, Cavaglieri RC, Khazim K, et al. Targeting NADPH oxidase with a novel dual Nox1/Nox4 inhibitor attenuates renal pathology in type 1 diabetes. Am J Physiol Renal Physiol. 2015;308: F1276-87.

97. Gray SP, Jha JC, Kennedy K, et al. Combined NOX1/4 inhibition with GKT137831 in mice provides dose-dependent reno- and atheroprotection even in established micro- and macrovascular disease. Diabetologia. 2017;60:927-37.

98. Cha JJ, Min HS, Kim KT, et al. APX-115, a first-in-class panNADPH oxidase (Nox) inhibitor, protects $\mathrm{db} / \mathrm{db}$ mice from renal injury. Lab Investig. 2017;97:419-31.

99. Chow F, Ozols E, Nikolic-Paterson DJ, Atkins RC, Tesch GH. Macrophages in mouse type 2 diabetic nephropathy: correlation with diabetic state and progressive renal injury. Kidney Int. 2004;65:116-28.

100. Kanamori H, Matsubara T, Mima A, et al. Inhibition of MCP-1/ CCR2 pathway ameliorates the development of diabetic nephropathy. Biochem Biophys Res Commun. 2007;360:772-7.

101. Menne J, Eulberg D, Beyer D, et al. C-C motif-ligand 2 inhibition with emapticap pegol (NOX-E36) in type 2 diabetic patients with albuminuria. Nephrol Dial Transplant. 2016;32:307-15.

102. de Zeeuw D, Bekker P, Henkel E, et al. The effect of CCR2 inhibitor CCX140-B on residual albuminuria in patients with type 2 diabetes and nephropathy: a randomised trial. Lancet Diabetes Endocrinol. 2015;3:687-96.

103. Montero RM, Covic A, Gnudi L, Goldsmith D. Diabetic nephropathy: what does the future hold? Int Urol Nephrol. 2016;48:99-113.

104. Meng XM, Huang XR, Xiao J, et al. Diverse roles of TGF-beta receptor II in renal fibrosis and inflammation in vivo and in vitro. $\mathrm{J}$ Pathol. 2012;227:175-88.

105. Ding Y, Kim JK, Kim SI, et al. TGF- $\beta 1$ protects against mesangial cell apoptosis via induction of autophagy. J Biol Chem. 2010;285: 37909-19.

106. McGaraughty S, Davis-Taber RA, Zhu CZ, et al. Targeting anti-TGF-beta therapy to fibrotic kidneys with a dual specificity antibody approach. J Am Soc Nephrol 2017; epub ahead of print.

107. Norlin J, Nielsen Fink L, Helding Kvist P, Douglas Galsgaard E, Coppieters K. Abatacept treatment does not preserve renal function in the streptozocin-induced model of diabetic nephropathy. PLoS One. 2016;11:e0152315.

108. Navarro-Gonzalez JF, Mora-Fernandez C, Muros de Fuentes M, et al. Effect of pentoxifylline on renal function and urinary albumin excretion in patients with diabetic kidney disease: the PREDIAN trial. J Am Soc Nephrol. 2015;26:220-9.

109. RamachandraRao SP, Zhu Y, Ravasi T, et al. Pirfenidone is renoprotective in diabetic kidney disease. J Am Soc Nephrol. 2009;20:1765-75.

110. Sharma K, Ix JH, Mathew AV, et al. Pirfenidone for diabetic nephropathy. J Am Soc Nephrol. 2011;22:1144-51.

111. Adler SG, Schwartz S, Williams ME, et al. Phase 1 study of antiCTGF monoclonal antibody in patients with diabetes and microalbuminuria. Clin J Am Soc Nephrol. 2010;5:1420-8.

112. Kanasaki K, Shi S, Kanasaki M, et al. Linagliptin-mediated DPP-4 inhibition ameliorates kidney fibrosis in streptozotocin-induced diabetic mice by inhibiting endothelial-to-mesenchymal transition in a therapeutic regimen. Diabetes. 2014;63:2120-31.

113. Roxburgh SA, Kattla JJ, Curran SP, et al. Allelic depletion of grem1 attenuates diabetic kidney disease. Diabetes. 2009;58: 1641-50.

114. Zhang Q, Shi Y, Wada J, et al. In vivo delivery of gremlin siRNA plasmid reveals therapeutic potential against diabetic nephropathy by recovering bone morphogenetic protein-7. PLoS One. 2010;5: e11709.

115. Church RH, Ali I, Tate M, et al. Gremlin1 plays a key role in kidney development and renal fibrosis. Am J Physiol Renal Physiol. 2017;312:F1141-57.

116. Nugent MM, Lee K, He JC. HIPK2 is a new drug target for antifibrosis therapy in kidney disease. Front Physiol. 2015;6:132.

117. Ka SM, Yeh YC, Huang XR, et al. Kidney-targeting Smad7 gene transfer inhibits renal TGF- $\beta /$ MAD homologue (SMAD) and nuclear factor $\mathrm{kB}(\mathrm{NF}-\mathrm{kB})$ signalling pathways, and improves diabetic nephropathy in mice. Diabetologia. 2012;55:509-19.

118. Putta S, Lanting L, Sun G, Lawson G, Kato M, Natarajan R. Inhibiting microRNA-192 ameliorates renal fibrosis in diabetic nephropathy. J Am Soc Nephrol. 2012;23:458-69.

119. Bai X, Geng J, Zhou Z, Tian J, Li X. MicroRNA-130b improves renal tubulointerstitial fibrosis via repression of snail-induced epithelial-mesenchymal transition in diabetic nephropathy. Sci Rep. 2016;6:20475.

120. Zhang Z, Sun L, Wang Y, et al. Renoprotective role of the vitamin D receptor in diabetic nephropathy. Kidney Int. 2008;73:163-71.

121. Tian Y, Lv G, Yang Y, et al. Effects of vitamin D on renal fibrosis in diabetic nephropathy model rats. Int J Clin Exp Pathol. 2014;7: 3028-37.

122. Ding Y, Choi ME. Autophagy in diabetic nephropathy. J Endocrinol. 2015;224:R15-30.

123. Kroemer G, Marino G, Levine B. Autophagy and the integrated stress response. Mol Cell. 2010;40:280-93.

124. Li H, Peng X, Wang Y, et al. Atg5-mediated autophagy deficiency in proximal tubules promotes cell cycle $\mathrm{G} 2 / \mathrm{M}$ arrest and renal fibrosis. Autophagy. 2016;12:1472-86.

125. Inoki $\mathrm{K}$, Mori $\mathrm{H}$, Wang $\mathrm{J}$, et al. mTORC1 activation in podocytes is a critical step in the development of diabetic nephropathy in mice. J Clin Invest. 2011;121:2181-96.

126. Hasegawa K, Wakino S, Simic P, et al. Renal tubular Sirt1 attenuates diabetic albuminuria by epigenetically suppressing Claudin1 overexpression in podocytes. Nat Med. 2013;19:1496-504.

127. Pagtalunan ME, Miller PL, Jumping-Eagle S, et al. Podocyte loss and progressive glomerular injury in type II diabetes. J Clin Invest. 1997;99:342-8.

128. Tagawa A, Yasuda M, Kume S, et al. Impaired podocyte autophagy exacerbates proteinuria in diabetic nephropathy. Diabetes. 2016;65:755-67.

129. Miaomiao W, Chunhua L, Xiaochen Z, Xiaoniao C, Hongli L, Zhuo Y. Autophagy is involved in regulating VEGF during high-glucose-induced podocyte injury. Mol BioSyst. 2016;12: 2202-12.

130. Kitada M, Takeda A, Nagai T, Ito H, Kanasaki K, Koya D. Dietary restriction ameliorates diabetic nephropathy through antiinflammatory effects and regulation of the autophagy via restoration of Sirt1 in diabetic Wistar fatty (fa/fa) rats: a model of type 2 diabetes. Exp Diabetes Res. 2011;2011:908185.

131. Kitada M, Ogura Y, Suzuki T, et al. A very-low-protein diet ameliorates advanced diabetic nephropathy through autophagy induction by suppression of the mTORC1 pathway in Wistar fatty rats, an animal model of type 2 diabetes and obesity. Diabetologia. 2016;59:1307-17.

132. Cho K, Chung JY, Cho SK, et al. Antihyperglycemic mechanism of metformin occurs via the AMPK/LXRalpha/POMC pathway. Sci Rep. 2015;5:8145.

133. Chen J, Zhao D, Zhu M, et al. Paeoniflorin ameliorates AGEsinduced mesangial cell injury through inhibiting RAGE/mTOR/ autophagy pathway. Biomed Pharmacother. 2017;89:1362-9.

134. Kim MY, Lim JH, Youn HH, et al. Resveratrol prevents renal lipotoxicity and inhibits mesangial cell glucotoxicity in a manner dependent on the AMPK-SIRT1-PGC1alpha axis in $\mathrm{db} / \mathrm{db}$ mice. Diabetologia. 2013;56:204-17. 
135. Lee MJ, Feliers D, Mariappan MM, et al. A role for AMPactivated protein kinase in diabetes-induced renal hypertrophy. Am J Physiol Renal Physiol. 2007;292:F617-27.

136. Sakaguchi M, Isono M, Isshiki K, Sugimoto T, Koya D, Kashiwagi A. Inhibition of mTOR signaling with rapamycin attenuates renal hypertrophy in the early diabetic mice. Biochem Biophys Res Commun. 2006;340:296-301.

137. Yang Y, Wang J, Qin L, et al. Rapamycin prevents early steps of the development of diabetic nephropathy in rats. Am J Nephrol. 2007;27:495-502.

138. Xia Y, Nivet E, Sancho-Martinez I, et al. Directed differentiation of human pluripotent cells to ureteric bud kidney progenitor-like cells. Nat Cell Biol. 2013;15:1507-15.

139. Taguchi A, Kaku Y, Ohmori T, et al. Redefining the in vivo origin of metanephric nephron progenitors enables generation of complex kidney structures from pluripotent stem cells. Cell Stem Cell. 2014;14:53-67.

140. Lam AQ, Freedman BS, Morizane R, Lerou PH, Valerius MT, Bonventre JV. Rapid and efficient differentiation of human pluripotent stem cells into intermediate mesoderm that forms tubules expressing kidney proximal tubular markers. J Am Soc Nephrol. 2014;25:1211-25.

141. Wang S, Li Y, Zhao J, Zhang J, Huang Y. Mesenchymal stem cells ameliorate podocyte injury and proteinuria in a type 1 diabetic nephropathy rat model. Biol Blood Marrow Transplant. 2013;19: 538-46.

142. Ezquer FE, Ezquer ME, Parrau DB, Carpio D, Yanez AJ, Conget PA. Systemic administration of multipotent mesenchymal stromal cells reverts hyperglycemia and prevents nephropathy in type 1 diabetic mice. Biol Blood Marrow Transplant. 2008;14:631-40.

143. Lv S, Liu G, Sun A, et al. Mesenchymal stem cells ameliorate diabetic glomerular fibrosis in vivo and in vitro by inhibiting TGF-beta signalling via secretion of bone morphogenetic protein 7. Diab Vasc Dis Res. 2014;11:251-61.

144. Nagaishi K, Mizue Y, Chikenji T, et al. Mesenchymal stem cell therapy ameliorates diabetic nephropathy via the paracrine effect of renal trophic factors including exosomes. Sci Rep. 2016;6: 34842 . 